\title{
Testing Familial Transmission of Smoking With Two Different Research Designs
}

\author{
Jorien L. Treur PhD', Karin J.H. Verweij PhD ${ }^{1,2}$, Abdel Abdellaoui PhD², \\ Iryna O. Fedko PhD', Eveline L. de Zeeuw PhD², Erik A. Ehli PhD' ${ }^{2,3}$, \\ Gareth E. Davies PhD ${ }^{2,3}$, Jouke-Jan Hottenga PhD², Gonneke Willemsen PhD², \\ Dorret I. Boomsma PhD², Jacqueline M. Vink ${ }^{1}$
}

${ }^{1}$ Radboud University Nijmegen, Behavioural Science Institute, the Netherlands; ${ }^{2}$ Department of Biological Psychology, Vrije Universiteit Amsterdam, the Netherlands; ${ }^{3}$ Avera Institute for Human Genetics, Sioux Falls, SD

Corresponding Author: Jorien L. Treur, PhD, Radboud University Nijmegen, Behavioural Science Institute, the Netherlands; E-mail: j.treur@bsi.ru.nl

\begin{abstract}
Introduction: Classical twin studies show that smoking is heritable. To determine if shared family environment plays a role in addition to genetic factors, and if they interact $(G \times E)$, we use a children-of-twins design. In a second sample, we measure genetic influence with polygenic risk scores (PRS) and environmental influence with a question on exposure to smoking during childhood.

Methods: Data on smoking initiation were available for 723 children of 712 twins from the Netherlands Twin Register (64.9\% female, median birth year 1985). Children were grouped in ascending order of risk, based on smoking status and zygosity of their twin-parent and his/her co-twin: never smoking twin-parent with a never smoking co-twin; never smoking twin-parent with a smoking dizygotic co-twin; never smoking twin-parent with a smoking monozygotic cotwin; and smoking twin-parent with a smoking or never smoking co-twin. For 4072 participants from the Netherlands Twin Register (67.3\% female, median birth year 1973), PRS for smoking were computed and smoking initiation, smoking heaviness, and exposure to smoking during childhood were available.

Results: Patterns of smoking initiation in the four group children-of-twins design suggested shared familial influences in addition to genetic factors. PRS for ever smoking were associated with smoking initiation in all individuals. PRS for smoking heaviness were associated with smoking heaviness in individuals exposed to smoking during childhood, but not in non-exposed individuals.

Conclusions: Shared family environment influences smoking, over and above genetic factors. Genetic risk of smoking heaviness was only important for individuals exposed to smoking during childhood, versus those not exposed (G×E).

Implications: This study adds to the very few existing children-of-twins (CoT) studies on smoking and combines a CoT design with a second research design that utilizes polygenic risk scores and data on exposure to smoking during childhood. The results show that shared family environment affects smoking behavior over and above genetic factors. There was also evidence for gene-environment interaction $(\mathrm{G} \times \mathrm{E})$ such that genetic risk of heavy versus light smoking was only important for individuals who were also exposed to (second-hand) smoking during childhood. Together, these findings give additional incentive to recommending parents not to expose their children to cigarette smoking.
\end{abstract}




\section{Introduction}

A large body of classical twin studies has shown that smoking behavior is moderately to highly heritable. ${ }^{1}$ In Dutch twins, individual differences in smoking initiation, whether or not a person ever smoked regularly in their lifetime, were explained by genetic factors for $44 \%$. The remaining variance was due to common environmental $(51 \%)$ and unique environmental factors $(5 \%)$. For nicotine dependence, heritability was higher at $75 \%$, with the remaining variance being explained by unique environmental factors. ${ }^{2}$

The objective of the present study was twofold: to explore the influence of being exposed to smoking in the family environment, over and above genetic factors, and to explore the interaction between such exposure to smoking and genetic factors. We utilize two research designs that have thus far rarely been applied to data on smoking. First the children-of-twins (CoT) design, which employs data of twins and their children. While monozygotic (MZ) twins share $100 \%$ of their DNA, dizygotic (DZ) twins have $50 \%$ of their segregating genes in common. The child of a MZ twin is genetically just as related to their own parent as to the MZ co-twin of their parent (their aunt or uncle). For the child of a DZ twin, the genetic relatedness to their parent's co-twin is half as large. This information allows us to categorize children of twins based on genetic and environmental risk. Children raised by a (MZ or DZ) twin who smokes, experience a high genetic and familial environmental risk of smoking. In contrast, children who are raised by a non-smoking twin who has a smoking MZ co-twin are at the same (high) genetic risk of smoking but at reduced environmental risk. Children raised by a non-smoking twin who has a smoking DZ co-twin are at intermediate genetic risk and reduced environmental risk. Finally, children of a non-smoking twin with a non-smoking (MZ or DZ) co-twin are at reduced genetic and reduced environmental risk. By comparing smoking behavior between these four categories of children of twins, we explore the effect of family environment, on top of genetic factors, on smoking. An indication of gene-environment interaction $(\mathrm{G} \times \mathrm{E})$ can also be obtained. ${ }^{3,4}$ In twin studies, the unique environment (not shared with other family members) is abbreviated with an "E" and the shared family environment with a "C" (for common environment). In the term " $\mathrm{G} \times \mathrm{E}$," the " $\mathrm{E}$ " refers to environment without a specification whether this is unique or shared. In the present study, $G \times E$ reflects an interaction between genes and the shared family environment.

Few studies applied the CoT design to data on smoking. In 1919 children of 1107 male twins and 1023 of their spouses from the United States, being exposed to a smoking parent increased the risk of nicotine dependence most, suggesting only an influence of shared family environment. ${ }^{5}$ In an earlier study from the same research group, both genetic and familial environmental influences on nicotine dependence were found. ${ }^{6}$ For smoking initiation and for being a current smoker, neither study found evidence for genetic or familial environmental transmission of smoking. The authors suggest that the latter is due to their correction for an extensive amount of covariates explaining most of the variance in smoking (parenting practices, parental psychiatric disorders, and sibling and peer smoking). In a study that included twins and their parents, parent-offspring resemblance in smoking behavior was accounted for completely by their genetic relatedness suggesting that familial environmental influences do not increase the risk of smoking. ${ }^{7}$ In the present study, we analyze data on smoking in a Dutch CoT sample.

With the CoT design, family structure is utilized to estimate the influence of genetic/environmental factors, without actually specifying these. It is assumed that having a smoking parent equals being exposed to smoking. However, it can be that the home environment reflects more than just exposure to smoking (eg, parental rules, attitudes ${ }^{8,9}$ ) and even if a parent smoked it does not automatically mean that the child was exposed if the parent only smoked outside the home. ${ }^{10}$ In a second research design, we therefore specify genetic and environmental factors for smoking. A polygenic risk score (PRS) is calculated for smoking behavior, based on risk variants detected in a large genomewide association meta-analysis. ${ }^{11}$ A PRS for an individual is the sum of multiple genetic variants across the genome, weighted by their effect size on the trait of interest. ${ }^{12}$ The PRS is expected to predict smoking behavior. One factor of risk in the family environment is exposure to second-hand smoking. We measured this by asking if a person was exposed to smoking during childhood in their home situation. By analyzing the PRS (genetic risk) together with exposure to smoking during childhood (family environment), we test their individual effect on smoking behavior, as well as their interaction.

The present study utilizes two research designs to assess genetic and familial environmental influences on smoking: (1) in a CoT design, including 723 children of 712 twins, the influence of genetic factors, environmental factors, and their interaction on smoking initiation are investigated and (2) in 4072 adults, the influence of PRS on smoking, exposure to smoking during childhood, and their interaction on smoking initiation and smoking heaviness are investigated.

\section{Methods}

\section{CoT Design}

The Netherlands Twin Register (NTR) is an ongoing cohort study that originated in 1987 at the Vrije Universiteit Amsterdam. Since 1991, surveys have been sent to adult participants every 2 or 3 years $^{13}$ and to adolescent participants at specific ages $(14,16$, and 18 years). ${ }^{14}$

We applied the CoT design to data on smoking initiation, a variable reflecting whether someone had ever initiated regular smoking in their lifetime, available in all NTR surveys. For 712 twins and their 723 children $(64.9 \%$ female, median year of birth 1985 , range 1949 to 1999 ), smoking data were available for one or more surveys. For 634 of these children of twins, data on smoking of the spouse of the twin, their other biological parent, were also available. In each survey, participants were first classified as current smokers, former smokers, or never-smokers. This classification was based on the questions "Have you ever smoked?" (answer categories "No," "A few times just to try," and "Yes") and "How often do you smoke now?" ("I don't smoke regularly," "I’ve quit smoking," "Once a week or less," "A few times a week," and "Once a day or more”). Current smokers were participants who had ever smoked and were currently smoking once a week or more. Former smokers were those who replied "I've quit smoking" to the second question. When the answers to these main questions where contradictory, or one of the two was missing, follow-up questions were utilized (for example, a valid answer to the question "How many cigarettes a day/a week do you smoke on average?" resulted in a classification as current smoker). Next, a variable reflecting smoking initiation was created. For a person who had never been classified as a current or former smoker, smoking initiation was coded as "No," while for a person who was a current or former smoker in one or more of the surveys smoking initiation was coded as "Yes." 
Genetic correlations between members of a family consisting of twins, their spouses, and their children are given in Supplemental Figure $1 . \mathrm{MZ}$ twins share $100 \%$ of their DNA while DZ twins share $50 \%$ of their segregating genes. A child of twin 1 is genetically just as related $(r g=0.5)$ to their own parent, twin 1 , as to their aunt or uncle, twin 2, if the twin pair is MZ. If the twin pair is DZ, the genetic relatedness between the child of twin 1 and twin 2 is twice as small $(r g=0.25)$. As in other CoT studies on smoking ${ }^{5,6}$ and alcohol, ${ }^{3,15}$ we grouped children of twins into four categories of risk (see Table 1). This approach is especially well suited for a dichotomous phenotype such as smoking initiation (never vs. ever smoking). ${ }^{4}$ In the lowest risk group are children of never smoking twins with a never smoking MZ/ DZ co-twin (risk group 0). Next are children of never smoking twins who have an ever smoking DZ co-twin (risk group 1) and children of never smoking twins who have an ever smoking MZ co-twin (risk group 2). At highest risk are children of ever smoking twins who have a never smoking or ever smoking MZ/DZ co-twin (risk group 3).

To test genetic and familial environmental influences on smoking, multivariate logistic regression analysis was performed. Smoking initiation of the children of twins was the dependent variable $(0=$ never smoking, 1 = ever smoking) and independent variables were risk group status (three dummy variables with risk group 0 as reference category), year of birth of the children and the twin-parent (continuous), and sex of the children and the twin-parent and their co-twin $(0=$ male, 1 = female). In a second regression analysis, the same independent variables were included plus smoking initiation of the spouse of the twin, the other biological parent of the child. Spousal data were not available for all families, and adding them reduced the sample from 723 to 634 children of twins, so we report results from both regression analyses. Family clustering was corrected for by employing the robust cluster option in STATA which utilizes information on family relatedness to adjust for correlation within families (ie, clusters). When comparing the four groups of children, the following rules were adopted: a pattern where smoking prevalence in risk group(s) $3>2,1,0$ suggests familial environmental effects; smoking prevalence risk group(s) 2, 3>1>0 suggests genetic effects; and smoking prevalence risk group(s) $3>2,1>0$ suggests $\mathrm{G} \times \mathrm{E} .{ }^{15}$

\section{Polygenic Risk Scores and Exposure to Smoking During Childhood}

For 4072 twin and non-twin participants of the NTR $167.3 \%$ female, median year of birth 1973, range 1939-1996) data on smoking behavior and genotype data were available.

\section{Smoking Behavior}

A question on exposure to smoking during childhood was included in the 10th NTR survey, sent to all adult participants in 2013/2014.
Participants were asked "Have you ever been exposed (at length) to cigarette smoke at home when you were a child (up to age 18) because other people smoked in your presence when you didn't smoke yourself?" Answer categories were "No" $(0)$ and "Yes." Information on smoking initiation $(0=$ never-smoker, $1=$ eversmoker) and smoking heaviness (number of cigarettes smoked per day) was also available. The number of cigarettes smoked per day now for current smokers or in the past for former smokers was asked. A variable was then created reflecting heavy ( $>20$ cigarettes per day) versus light ( $\leq 10$ cigarettes per day) smoking to provide a rigorous contrast in smoking heaviness as was done previously in a large review paper. ${ }^{16}$

\section{Genotype Data}

Single-nucleotide polymorphism (SNP) data were available from genome-wide SNP arrays, collected within the NTR through several projects between 2004 and 2008. ${ }^{17,18}$ Further details on genotyping and quality control are provided in the supplemental text. A PRS reflects a summation of genetic risk from multiple genetic variants genomewide, weighted on their effect size. ${ }^{12}$ To obtain effect sizes for each genetic variant, summary statistics from genomewide association study for smoking initiation and number of cigarettes smoked per day were utilized. ${ }^{11}$ A novel method, LDpred, was employed to compute PRS. ${ }^{19}$ As far as we know, there are no published studies to date utilizing PRS for smoking behavior with this method. LDpred computes SNP weights based on their effect size estimates, their LD with other SNPs, and the degree of polygenicity of the trait, quantified as the expected fraction of causal SNPs. The LD structure was based on the European populations from the 1000 Genomes dataset. PRS were calculated for several assumed fractions of causal genetic markers: $0.01 \%$, $0.03 \%, 0.1 \%, 0.3 \%, 1 \%, 3 \%, 10 \%$, and $100 \%$. For a more elaborate explanation of this method of calculating PRS we refer to Vilhjalmsson et al. ${ }^{19}$. Computed PRS were transformed into Z-values before analysis.

With logistic regression analysis the association between exposure to smoking during childhood and smoking initiation/smoking heaviness was first tested, as was the association between the PRS and their respective smoking phenotypes. In a second step, exposure to smoking during childhood and PRS were analyzed together to assess their effect when corrected for each other. Finally, regression analyses between the PRS and their respective smoking phenotypes were stratified on exposure to smoking during childhood (ie, in exposed vs. non-exposed separately) to test for $\mathrm{G} \times \mathrm{E}$. All analyses were corrected for sex, year of birth, and the top 10 principle components reflecting population structure to correct for possible population stratification. ${ }^{20}$ Family clustering was corrected for with the robust cluster option in STATA.

Table 1. Four group CoT design

Children's level of smoking risk

\begin{tabular}{|c|c|c|c|c|c|}
\hline & $\begin{array}{l}\text { Phenotype } \\
\text { twin-parent }\end{array}$ & $\begin{array}{c}\text { Phenotype } \\
\text { co-twin of parent }\end{array}$ & Genetic risk & $\begin{array}{c}\text { Familial } \\
\text { environmental risk }\end{array}$ & $\begin{array}{c}\text { Genotype } \times \text { environmental } \\
\text { interaction }\end{array}$ \\
\hline Risk group 0 & Never-smoker & Never-smoker, MZ/DZ twin & Low & Low & Very low \\
\hline Risk group 1 & Never-smoker & Ever-smoker, DZ twin & Intermediate & Low & Low \\
\hline Risk group 2 & Never-smoker & Ever-smoker, $\mathrm{MZ}$ twin & High & Low & Low \\
\hline Risk group 3 & Ever-smoker & Never or ever-smoker, MZ/DZ twin & High & High & High \\
\hline
\end{tabular}

$\mathrm{CoT}=$ children-of-twins; $\mathrm{MZ}=$ monozygotic, $\mathrm{DZ}=$ dizygotic. 


\section{Results}

\section{CoT Design}

Of the 723 children of twins, $202(27.9 \%)$ had initiated smoking. The first regression analysis in Table 2 shows that children in risk groups 2 and 3 were at significantly higher odds of smoking initiation compared to children in risk group 0 (odds ratio [OR] 2.43 $95 \%$ CI 1.05 to 5.66 and 2.45 CI 1.52 to 3.94 , respectively). In the second regression analysis, corrected for smoking status of the spouse of the twin, only the difference between risk group 3 and risk group 0 remained significant (OR 2.42 CI 1.47 to 3.98). The latter demonstrates that having a smoking (twin-)parent results in the highest odds of smoking. In the first regression analysis, having a smoking aunt/uncle who is genetically identical to one's parent increased the odds of smoking too. In both regression analyses, the odds of smoking for children with a smoking (MZ co-twin-) aunt/uncle did not differ significantly when compared to the odds of smoking for children with a smoking (twin-)parent (risk group 2 compared to risk group 3 OR 0.99 CI 0.47 to 2.12 and OR $0.78 \mathrm{CI}$ 0.33 to 1.80 , respectively). Together, these findings imply that shared family environment and, to a lesser extent, genetic factors influence smoking initiation.

In the case of an interaction between genetic and environmental factors, risk group 3 would show the highest smoking prevalence, followed by risk groups 2 and 1 together, which in turn would show a higher prevalence than risk group 0 . For regression analysis 2 especially, there seemed to be such a pattern. However, the odds of smoking were not significantly higher for risk groups 1 and 2 compared to risk group 0 . This was also not the case when these two groups were combined. There is thus no strong evidence for $\mathrm{G} \times \mathrm{E}$ interaction for smoking initiation, possibly due to a lack of power.

\section{Polygenic Risk Scores and Exposure to Smoking During Childhood}

Of the 4072 participants, 1489 (36.6\%) had initiated smoking. For 1426 of those smokers, information on number of cigarettes smoked per day was available $(M=15.3$, standard deviation $=9.2)$. After dichotomizing this variable, ${ }^{16}$ there were 275 heavy smokers $(>20$ cigarettes per day) and 538 light smokers ( $\leq 10$ cigarettes per day). The odds of smoking initiation were higher for those exposed to smoking during childhood versus those not exposed (OR 1.69 CI 1.47 to 1.95 ) as were the odds of being a heavy smoker versus a light smoker (OR 1.65 CI 1.18 to 2.32). A possible limitation of measuring childhood exposure to smoking with a survey question is differential recall bias (smokers having a higher propensity to remember smoking in their family than non-smokers). In 333 discordant twin pairs, where one twin initiated smoking and the other did not, we found no significant difference in their answer to the question on smoking exposure during childhood ( $\chi^{2} p$-value 0.321$)$, suggesting that there was no such recall bias. PRS were computed for the following fractions of causal markers: $0.01 \%, 0.03 \%, 0.1 \%$, $0.3 \%, 1 \%, 3 \%, 10 \%$, and $100 \% .{ }^{24}$ PRS for smoking initiation significantly predicted smoking initiation for the highest five of these fractions (ORs 1.08-1.12, $R^{2} 0.1-0.3 \%$, Supplementary Table 1). PRS for smoking heaviness significantly predicted heavy versus light smoking for the highest six fractions (ORs 1.17-1.26, $R^{2} 0.7-1.5 \%$, Supplementary Table 2). The ORs here represent the odds of smoking initiation/heavy versus light smoking for each standard deviation increase in the respective PRS. When analyzing exposure to smoking during childhood and PRS together, thus correcting them for each other, both were still associated with smoking behavior (Supplementary Tables 3 and 4). This implies an added effect of exposure to smoking during childhood, in addition to genetic risk. In further analyses, we continued with fractions $0.1 \%$ to $10 \%$, as these were the most predictive.

The association between PRS for smoking initiation and smoking initiation and between PRS for smoking heaviness and heavy versus light smoking are shown for the total group and stratified on exposure to smoking during childhood in Figures 1 and 2, respectively. For smoking initiation, the association with PRS was very similar in individuals who were not exposed to smoking during childhood to the association in those who were exposed. In contrast, heaviness of smoking PRS significantly predicted heavy versus light smoking in individuals who were exposed to smoking during childhood (ORs 1.24-1.38, $R^{2} 1.4-3.0 \%$ ), but not in individuals who were not exposed (ORs 1.06-1.11, $R^{2} 0.1-0.2 \%$ ). This implies an interaction between these two factors. When analyzing the total sample and including an interaction term between exposure to smoking during childhood and PRS, there was no evidence for an interaction in the case of smoking initiation and borderline significant results providing weak evidence for a positive interaction in the case of smoking heaviness (Supplementary Tables 7 and 8).

\section{Discussion}

The present study employed two research designs to assess familial transmission of smoking behavior. With a CoT design, there was evidence for familial environmental influence on smoking initiation in addition to genetic risk, while statistical power was too low to test gene environment interaction $(\mathrm{G} \times \mathrm{E})$. When specifying genetic risk with a PRS and environmental influence with a question on exposure to smoking during childhood, there was also evidence for an added influence of familial environment but no evidence for $\mathrm{G} \times \mathrm{E}$. For smoking heaviness, there was some evidence for $G \times E$ such that PRS for smoking heaviness were only associated with smoking heaviness when participants were exposed to smoking during childhood, not when participants were not exposed.

Our findings are consistent with a large body of literature in showing that both shared family environment and genetic factors influence whether someone will smoke. ${ }^{1,2}$ Previous CoT studies on smoking found evidence for an influence of the shared family environment in addition to genetic effects for nicotine dependence, but not for smoking initiation. 5,6 This discrepancy may be due to the fact that in these previous studies, a large amount of (psychiatric) covariates were taken into account. When we analyzed PRS for smoking initiation together with a variable reflecting exposure to smoking during childhood, both were significantly associated with smoking initiation. This confirms the effect of genetic influences on smoking initiation but also implies an effect of the shared family environment in addition to genetic risk. Neither of the research designs showed evidence for an interaction between genetic risk and shared family environment in their influence on smoking initiation.

PRS for smoking heaviness as well as exposure to smoking during childhood were associated with heavy versus light smoking. When stratifying PRS smoking heaviness analyses, PRS for smoking heaviness only predicted heavy versus light smoking in individuals who were exposed to smoking during childhood, but not in individuals who were not exposed. Results were similar when analyzing a continuous variable of cigarettes per day instead of heavy versus light smoking (data not shown). Previous research demonstrated that 


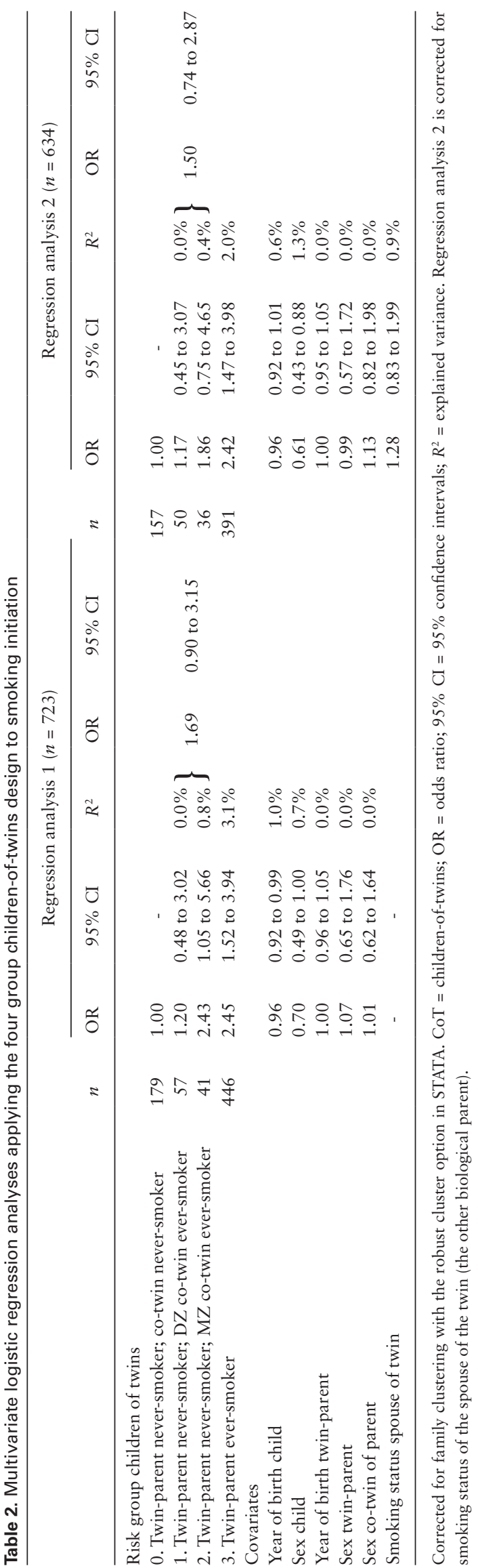

exposure to smoking during childhood influences a person's sensitivity to their first dose of nicotine if they initiate smoking. ${ }^{21}$ Sensitivity to nicotine in turn predicts whether or not a person becomes a heavy smoker. ${ }^{22,23}$ We now show that the influence of exposure to smoking during childhood on whether a person becomes a heavy smoker depends on that person's genetic make-up. So far, interaction between genetic factors and exposure to smoking has been tested with candidate genes. Although such studies do not match our PRS approach exactly, their results can illustrate what kinds of environmental factors may interact with genetic risk. In 171 young adolescents who had never smoked, there was no interaction between three reward-related candidate gene polymorphisms and exposure to smoking by parents, siblings, or peers on first time smoking. ${ }^{24}$ In a later study of 376 smoking adolescents, those who carried the DRD2 Tag1A A2-allele developed nicotine dependence more rapidly when exposed to parental smoking, compared to those not carrying this allele. Controversially, the A2-allele had previously been shown to make individuals less sensitive to the rewarding effects of nicotine. ${ }^{25}$ Finally, nicotine dependence was less affected by peer smoking in individuals with a high-risk genotype of a genetic variant in the CHRNA5 gene (rs16969968) than in individuals with a lower-risk genotype. ${ }^{26}$ These findings corroborate those from the present study in showing that genetic risk for heaviness of smoking can interact with exposure to smoking by parents, siblings, or peers.

The present study was especially novel in that PRS were utilized, reflecting genetic risk across the whole genome. In a similar design, Musci et al. ${ }^{27}$ found that PRS for smoking interacted with social environment in predicting the trajectory of tobacco use. Adolescents experiencing less parental monitoring, a higher proportion of substance-using friends, and high genetic risk were at highest odds of smoking. ${ }^{27}$ Meyers et al. ${ }^{28}$ tested interactions between PRS and experiencing "traumatic events" and "neighborhood social cohesion." They found that the association between PRS and smoking was stronger in those who experienced more traumatic life events while it was weaker in those who lived in a neighborhood with greater social cohesion. ${ }^{28}$ These and our findings support the value of PRS when testing $\mathrm{G} \times \mathrm{E}$, as highlighted by a recent commentary on future research on the genetics of addiction. ${ }^{29}$

Apart from an interaction between genetic and environmental factors, there may also be gene-environment correlation ( $r \mathrm{GE})$. In that case, genetic factors are directly correlated with the environmental variable in question. ${ }^{30}$ In the present study, we tested the association between PRS for smoking and exposure to smoking during childhood and found no evidence for an association and thus no evidence for gene-environment correlation (data not shown). This is contrary to what one might expect given that childhood exposure to smoking will often be the result of parental smoking and parents also pass along their (smoking risk) genes to their children. The lack of a gene-environment correlation could have to do with individual differences in parents' choices regarding exposing their children to smoking, with some smoking around their children and others not. In addition, exposure to smoking may also come from other family members such as grandparents or aunts/uncles.

An important limitation to the current study is that for the CoT design the number of included participants was relatively low. This resulted in low statistical power for the smaller risk groups, preventing us from definitively assessing $\mathrm{G} \times \mathrm{E}$. It also prevented us from correcting for a more elaborate set of covariates. We did correct for smoking status of the spouse of the twin (the other biological parent 


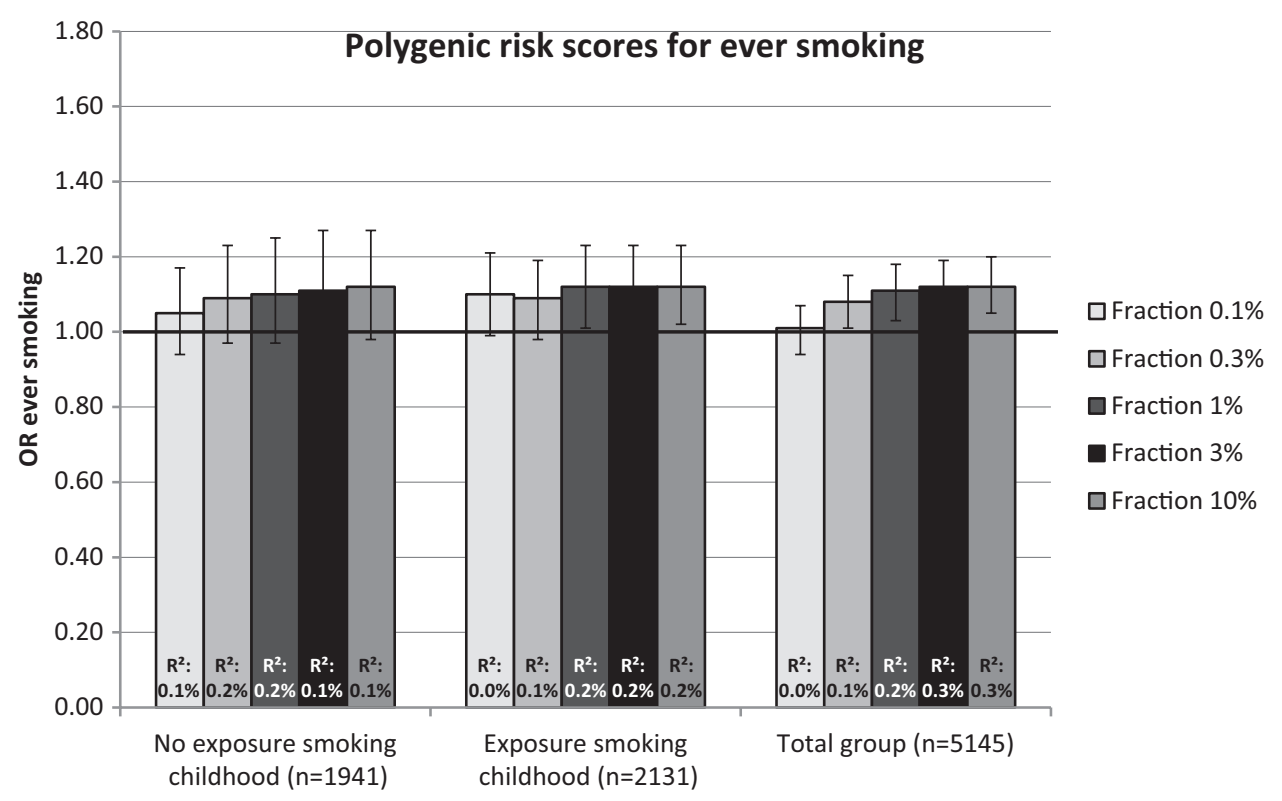

Figure 1. Odds ratios (OR) represent the odds of ever versus never smoking for each standard deviation (SD) increase in the polygenic risk score (PRS) for ever smoking, corrected for year of birth, sex, 10 principle components (PCs), and family clustering in STATA. PRS were calculated for varying fractions of genetic variants $(0.1 \%, 0.3 \%, 1 \%, 3 \%, 10 \%) . R^{2}$ : explained variance.

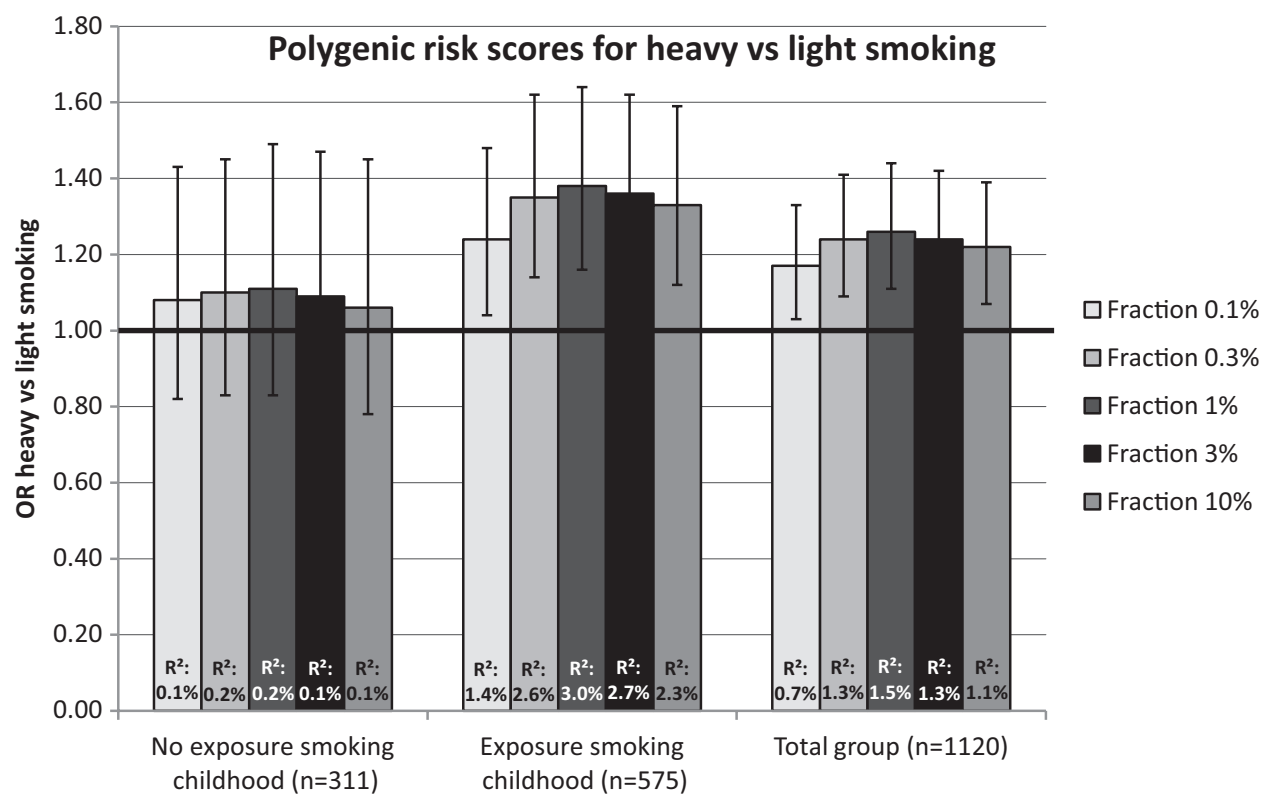

Figure 2. Odds ratios (OR) represent the odds of heavy (>20 cigarettes per day) versus light ( $\leq 10$ cigarettes per day) smoking for each standard deviation (SD) increase in the polygenic risk score (PRS) for smoking heaviness, corrected for year of birth, sex, 10 principle components (PCs), and family clustering in STATA. PRS were calculated for varying fractions of genetic variants $(0.1 \%, 0.3 \%, 1 \%, 3 \%, 10 \%)$. $R^{2}$ : explained variance.

of the child) thereby strengthening our findings. We propose that with larger numbers of twins and children of twins, it would be better to utilize smoking status of the spouse to create more risk groups (combinations of 0,1, 2 smoking parents and/or smoking MZ/DZ co-twins), instead of merely including it as a covariate. The smoking behavior of the spouse of the twin has an environmental and genetic influence on smoking of the child, just as much as the smoking behavior of the twin him/herself does. Such an approach would also take into account the fact that a child of a smoking twin with a smoking co-twin may be at higher genetic risk than a child of a smoking twin with a non-smoking co-twin.
Overall, this study showed the combined importance of genetic and familial environmental influences on smoking behavior. More specifically, we demonstrated that exposure to smoking in the family home has a unique effect, over and above genetic factors. For smoking heaviness, genetic risk only played a role for those individuals who were previously exposed to smoking during childhood. This could have important implications. It suggests that individuals who have been exposed to smoking in their childhood and who are genetically vulnerable to heavy smoking, are at higher risk of becoming a heavy smoker than are individuals who carry the same genetic risk variants but who were not exposed to smoking during childhood. 
The group that is genetically susceptible and exposed to smoking should therefore be particularly targeted with preventive measures. It also gives additional incentive to recommending parents not to expose their children to cigarette smoking.

\section{Funding}

This study was supported by the European Research Council (ERC) (Grant number 284167: "Beyond the Genetics of Addiction" [principal investigator JMV] and Grant number 230374: "Genetics of Mental Illness" [principal investigator DIB]) and by grants from the Netherlands Organization for Scientific Research (NWO): ZonMW Addiction (31160008 and NWO 016115-035), Genetic and Family Influences on Adolescent Psychopathology and Wellness (NWO 463-06-001), A twin-sib study of adolescent wellness (NWOVENI 451-04-034), Gravitation program of the Dutch Ministry of Education, Culture and Science and the Netherlands Organization for Scientific Research (NWO 0240-001-003), VU University's Institute for Health and Care Research (EMGO+) and Neuroscience Campus Amsterdam (NCA), and Biomolecular Resources Research Infrastructure (BBMRI-NL, 184.021.007). Genotyping was possible by grants from NWO/SPI 56-464-14192, the Genetic Association Information Network (GAIN) of the Foundation for the National Institutes of Health, Rutgers University Cell and DNA Repository (NIMH U24 MH068457-06), the Avera Institute, Sioux Falls, South Dakota (USA), and the National Institutes of Health (NIH R01 HD042157-01A1, MH081802, Grand Opportunity grants 1RC2 MH089951 and 1RC2 MH089995). KJHV is supported in part by a 2014 NARSAD Young Investigator Grant from the Brain \& Behavior Research Foundation. We would also like to acknowledge the work of the Tobacco and Genetics (TAG) consortium.

\section{Declaration of Interests}

None declared.

\section{References}

1. Kaprio J. Genetic epidemiology of smoking behavior and nicotine dependence. COPD. 2009;6(4):304-306.

2. Vink JM, Willemsen G, Boomsma DI. Heritability of smoking initiation and nicotine dependence. Behav Genet. 2005;35(4):397-406.

3. Heath AC, Nelson EC. Effects of the interaction between genotype and environment. Research into the genetic epidemiology of alcohol dependence. Alcohol Res Health. 2002;26(3):193-201.

4. McAdams TA, Neiderhiser JM, Rijsdijk FV, Narusyte J, Lichtenstein P, Eley TC. Accounting for genetic and environmental confounds in associations between parent and child characteristics: a systematic review of children-of-twins studies. Psychol Bull. 2014;140(4):1138-1173.

5. Scherrer JF, Xian H, Pan H, et al. Parent, sibling and peer influences on smoking initiation, regular smoking and nicotine dependence. Results from a genetically informative design. Addict Behav. 2012;37(3):240-247.

6. Xian H, Scherrer JF, Pergadia ML, et al. Contribution of parental psychopathology to offspring smoking and nicotine dependence in a genetically informative design. J Stud Alcohol Drugs. 2010;71(5):664-673.

7. Boomsma DI, Koopmans JR, Van Doornen LJ, Orlebeke JF. Genetic and social influences on starting to smoke: a study of Dutch adolescent twins and their parents. Addiction. 1994;89(2):219-226.

8. Otten R, Harakeh Z, Vermulst AA, Van den Eijnden RJ, Engels RC. Frequency and quality of parental communication as antecedents of adolescent smoking cognitions and smoking onset. Psychol Addict Behav. 2007;21(1):1-12.

9. Harakeh Z, Scholte RH, de Vries H, Engels RC. Parental rules and communication: their association with adolescent smoking. Addiction. 2005;100(6):862-870.
10. Orton S, Jones LL, Cooper S, Lewis S, Coleman T. Predictors of children's secondhand smoke exposure at home: a systematic review and narrative synthesis of the evidence. PLoS One. 2014;9(11):e112690.

11. Tobacco and Alcohol Genetics Consortium (TAG). Sequence variants at CHRNB3-CHRNA6 and CP2A6 affect smoking behaviour. Nat Genet. 2010;42(5):448-453.

12. Wray NR, Goddard ME, Visscher PM. Prediction of individual genetic risk to disease from genome-wide association studies. Genome Res. 2007;17(10):1520-1528.

13. Willemsen G, Vink JM, Abdellaoui A, et al. The Adult Netherlands Twin Register: twenty-five years of survey and biological data collection. Twin Res Hum Genet. 2013;16(1):271-281.

14. van Beijsterveldt CE, Groen-Blokhuis M, Hottenga JJ, et al. The Young Netherlands Twin Register (YNTR): longitudinal twin and family studies in over 70,000 children. Twin Res Hum Genet. 2013;16(1): 252-267.

15. Knopik VS, Jacob T, Haber JR, Swenson LP, Howell DN. Paternal alcoholism and offspring ADHD problems: a children of twins design. Twin Res Hum Genet. 2009;12(1):53-62.

16. Hartz SM, Short SE, Saccone NL, et al. Increased genetic vulnerability to smoking at CHRNA5 in early-onset smokers. Arch Gen Psychiatry. 2012;69(8):854-860.

17. Willemsen G, de Geus EJ, Bartels M, et al. The Netherlands Twin Register biobank: a resource for genetic epidemiological studies. Twin Res Hum Genet. 2010;13(3):231-245.

18. Nivard MG, Mbarek H, Hottenga JJ, et al. Further confirmation of the association between anxiety and CTNND2: replication in humans. Genes Brain Behav. 2014;13(2):195-201.

19. Vilhjalmsson B, Yang J, Hilary KF, et al. Modeling linkage disequilibrium increases accuracy of polygenic risk scores. Am J Hum Genet. 2015;97(4):576-592.

20. Abdellaoui A, Hottenga JJ, de Knijff P, et al. Population structure, migration, and diversifying selection in the Netherlands. Eur J Hum Genet. 2013;21(11):1277-1285.

21. Okoli CT, Richardson CG, Johnson JL. An examination of the relationship between adolescents' initial smoking experience and their exposure to peer and family member smoking. Addict Behav. 2008;33(9):1183-1191.

22. DiFranza JR, Savageau JA, Fletcher K, et al. Susceptibility to nicotine dependence: the Development and Assessment of Nicotine Dependence in Youth 2 study. Pediatrics. 2007;120(4):e974-e983.

23. Sartor CE, Lessov-Schlaggar CN, Scherrer JF, et al. Initial response to cigarettes predicts rate of progression to regular smoking: findings from an offspring-of-twins design. Addict Behav. 2010;35(8):771-778.

24. Schuck K, Otten R, Engels RC, Kleinjan M. Initial responses to the first dose of nicotine in novel smokers: the role of exposure to environmental smoking and genetic predisposition. Psychol Health. 2014;29(6):698-716.

25. Kleinjan M, Engels RC, DiFranza JR. Parental smoke exposure and the development of nicotine craving in adolescent novice smokers: the roles of DRD2, DRD4, and OPRM1 genotypes. BMC Pulm Med. 2015;15:115.

26. Johnson EO, Chen LS, Breslau N, et al. Peer smoking and the nicotinic receptor genes: an examination of genetic and environmental risks for nicotine dependence. Addiction. 2010;105(11):2014-2022.

27. Musci RJ, Uhl G, Maher B, Ialongo NS. Testing gene $\times$ environment moderation of tobacco and marijuana use trajectories in adolescence and young adulthood. J Consult Clin Psychol. 2015;83(5):866-874.

28. Meyers JL, Cerdá M, Galea S, et al. Interaction between polygenic risk for cigarette use and environmental exposures in the Detroit Neighborhood Health Study. Transl Psychiatry. 2013;3:e290.

29. Vink JM. Genetics of addiction: future focus on gene $\times$ environment interaction? J Stud Alcohol Drugs. 2016;77(5):684-687.

30. Do E, Maes H. Narrative review of genes, environment, and cigarettes. Ann Med. 2016;48(5):337-351. 